Virginia Commonwealth University vCU Scholars Compass

2007

\title{
Investigations on the structure of dimethyl sulfoxide and acetone in aqueous solution
}

Sylvia E. McLain

Oak Ridge National Laboratory

Alan K. Soper

Rutherford Appleton Laboratory

Alenka Luzar

Virginia Commonwealth University

Follow this and additional works at: http://scholarscompass.vcu.edu/chem_pubs

Part of the Chemistry Commons

McLain, S. E., Soper, A. K., Luzar, A. Investigations on the structure of dimethyl sulfoxide and acetone in aqueous solution. The Journal of Chemical Physics 127, 174515 (2007). Copyright (C 2007 AIP Publishing LLC.

\section{Downloaded from}

http://scholarscompass.vcu.edu/chem_pubs/62

This Article is brought to you for free and open access by the Dept. of Chemistry at VCU Scholars Compass. It has been accepted for inclusion in Chemistry Publications by an authorized administrator of VCU Scholars Compass. For more information, please contact libcompass@vcu.edu. 


\title{
Investigations on the structure of dimethyl sulfoxide and acetone in aqueous solution
}

\author{
Sylvia E. McLain ${ }^{\text {a) }}$ \\ Spallation Neutron Source and Center for Molecular Biophysics, Oak Ridge National Laboratory, \\ P.O. Box 2008, Oak Ridge, Tennessee 37831-6475, USA \\ Alan K. Soper \\ ISIS Facility, Rutherford Appleton Laboratory, Chilton, Didcot, Oxon OX11 0QX, United Kingdom \\ Alenka Luzar \\ Department of Chemistry, Virginia Commonwealth University, Richmond, Virginia 23284-2006, USA
}

(Received 27 July 2007; accepted 22 August 2007; published online 7 November 2007)

\begin{abstract}
Aqueous solutions of dimethyl sulfoxide (DMSO) and acetone have been investigated using neutron diffraction augmented with isotopic substitution and empirical potential structure refinement computer simulations. Each solute has been measured at two concentrations-1:20 and 1:2 solute:water mole ratios. At both concentrations for each solute, the tetrahedral hydrogen bonding network of water is largely unperturbed, though the total water molecule coordination number is reduced in the higher 1:2 concentrations. With higher concentrations of acetone, water tends to segregate into clusters, while in higher concentrations of DMSO the present study reconfirms that the structure of the liquid is dominated by DMSO-water interactions. This result may have implications for the highly nonideal behavior observed in the thermodynamic functions for 1:2 DMSO-water solutions. (C) 2007 American Institute of Physics. [DOI: 10.1063/1.2784555]
\end{abstract}

\section{INTRODUCTION}

The importance of understanding the balance between hydrophobic or hydrophilic solute-solvent interactions is of fundamental importance as these interactions drive many natural processes. Although there is no single model which can account for all of the properties of water, ${ }^{1}$ there have been considerable efforts to understand its properties as a solvent. Binary mixtures of simple organic molecules and water have been studied using a variety of techniques; ${ }^{2-21}$ as understanding binary systems is a necessary step toward understanding the solution properties of water itself. Here the solutes DMSO and acetone have been investigated in aqueous solution. DMSO and acetone, both small organic molecules, differ in chemical composition by only one atom, the central atom, which is sulfur in DMSO and carbon in acetone. Even though compositionally similar, DMSO and acetone have very different molecular geometries as a result of the lone electron pair on sulfur which is not present on the central carbon atom in acetone. Specifically, DMSO, which forms a trigonal pyramid [Fig. 1(a)], is in the Cs molecular point group while acetone, a trigonal planar molecule [Fig. 1(b)] shows the higher symmetry of the $C_{2 v}$ point group.

As a net liquid, acetone is polar $(\mu=2.88 \mathrm{D})$ and aprotic, and in-solution acetone can accept hydrogen bonds via its carbonyl oxygen. Previous work on the acetone-water system in the liquid phase indicates that the addition of acetone to water perturbs the water structure by disrupting the tetrahedral hydrogen bonding network present in bulk water,

\footnotetext{
a) Author to whom correspondence should be addressed. Electronic mail: mclainse@ornl.gov
}

namely, by lowering the number of hydrogen bonds present between water molecules. ${ }^{10,12-14,17,18}$ However, while some of these studies are thought to be indicative of clustering or segregation between the water molecules upon the addition of acetone, ${ }^{10,18}$ others are interpreted as having a random distribution of water and acetone molecules at any given concentration. ${ }^{13,14}$ The DMSO-water system, also a simple binary mixture, is similar to acetone in some respects. As a neat liquid, DMSO is also polar and aprotic and is a hydrogen bond acceptor via its carbonyl oxygen but does not donate hydrogen bonds. However, DMSO has much higher dipole moment at $3.96 \mathrm{D}$ and while both molecules possess a high dielectric constant (acetone $=20.7$ at $20{ }^{\circ} \mathrm{C}$; DMSO $=47.2$ at $20^{\circ} \mathrm{C}$ ), the dielectric constant of DMSO is much higher than that of acetone.

The dimethyl sulfoxide (DMSO) $\left[\left(\mathrm{CH}_{3}\right)_{2} \mathrm{SO}\right]$ water system is of particular importance as DMSO is used widely in industry, biology, organic chemistry, and pharmacology to name a few of its uses. ${ }^{22}$ In particular, DMSO has the ability to cross cellular membranes without causing degradation

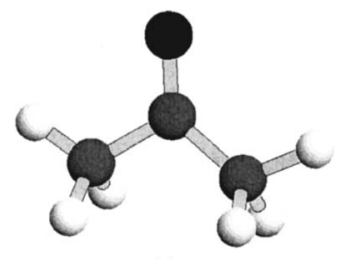

(a)

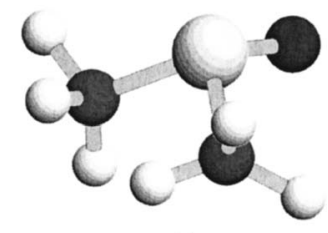

(b)
FIG. 1. Molecular structure of acetone (left panel) and DMSO (right panel). The white atoms are hydrogen, the gray atoms are carbon, the black atoms are oxygen, and the off-white atom in the center of the molecule in DMSO is sulfur. 
which is of premier importance in drug delivery. ${ }^{22}$ DMSO is also used as a cryoprotectant where at a DMSO:water ratio of $1: 2$ the freezing point of water is depressed to $\sim-70{ }^{\circ} \mathrm{C} .{ }^{23}$ In addition, other physical properties of the 1:2 DMSO-water solution indicate a pronounced nonideal behavior at this concentration such as large negative heats of mixing, ${ }^{24,25}$ and excess mixing volumes. ${ }^{26}$

Two previous neutron diffraction studies of the DMSOwater system have been reported..$^{21,27}$ In the first one, ${ }^{21}$ the structure of water in concentrated aqueous solutions of DMSO (1:2 DMSO in water) was investigated, using neutron diffraction with H/D isotope substitution on the water hydrogen atoms in order to separate out the water-H to water-H correlations. This concentration of DMSO in water is unique as there are several minima observed in many of the thermodynamic properties of the solution. ${ }^{24,28}$ The other concentration previously measured, a 1:4 DMSO to water molar ratio, allowed for the investigation of DMSO hydration as a function of increasing dilution. These data revealed that the peak locations in $g_{\mathrm{H}_{w} \mathrm{H}_{w}}(r)$ of the first molecular coordination shell were still preserved at both concentrations studied, when compared to pure water even though the water-water coordination number was reduced, indicating the local arrangement of water molecules remained largely intact in the presence of DMSO. The change in coordination number with concentration of the solute agreed with predictions by a mean-field hydrogen bonding model of DMSO-water solutions. ${ }^{25}$ The second neutron diffraction experiment ${ }^{27}$ revealed that the DMSO-water interaction through hydrogen bonding was stronger than the water-water hydrogen bonding. From these two studies, the molecular picture emerged that in the mixing process between water and DMSO, hydrogen bonding is simply transferred from water-water interactions to waterDMSO interactions in agreement with the qualitative ideas underlying the mean-field model of this system. ${ }^{25}$

Recently, a comparative study of DMSO and acetone as neat liquids was reported ${ }^{29}$ using neutron diffraction and empirical potential structure refinement (EPSR) modeling. Through a detailed analysis of the orientational correlations obtained from EPSR fits to measured neutron diffraction data, it was found that DMSO and acetone showed some similarities but also marked differences between their nearest-neighbor dipole alignments in the liquid. At short distances the dipoles of neighboring molecules were in antiparallel configuration, while at longer distances both molecules tended to be aligned as head to tail in the manner of dipole ordering.

The current report brings the comparative study of DMSO and acetone to their aqueous solutions. In order to reveal the structure in these solutions, a combination of neutron diffraction measurements and EPSR modeling have been performed. Both DMSO and acetone in water at two different concentrations-1:20 solute:water and 1:2 solute:water-have been investigated in this study, where these concentrations represent two practical limits of the concentration scale. The 1:20 concentration was chosen as it represents a realistically dilute solution, while still ensuring that there is sufficient contrast to give rise to a measurable diffraction signal from the solute, which is not overwhelmed by the bulk water signal. At the other end of the scale, the 1:2 solute to water concentration was chosen because, as described above, this is a unique concentration for the thermodynamic properties of DMSO in solution. Acetone was measured under the same conditions as DMSO in order to establish the differences these contrasting molecular structures have on the surrounding water environment.

While most of the data in the present work have not previously been published, the 1:2 DMSO:water data have been previously reported. ${ }^{27}$ Since the publication of the first study, a number of changes have occurred in the EPSR modeling method. In particular, instead of fitting the composite radial distribution functions in $r$ space as was done previously, the total differential cross section diffraction data are now fit in reciprocal space, ${ }^{30,31}$ which gives a more faithful account of fitting uncertainties in the data interpretation procedures. As a result all the data shown here have been analyzed or reanalyzed again from raw neutron counts. As will be seen below, this gives a somewhat different account of the local order in these solutions compared to what was extracted previously, ${ }^{27}$ although these are often quantitative rather qualitative changes. What is important in the present study is the comparison between acetone and DMSO in water measured at the same molar concentrations. The differences between the present results and the earlier conclusions from the 1:2 DMSO:water study ${ }^{27}$ are noted in the text.

\section{NEUTRON DIFFRACTION MEASUREMENTS}

Neutron diffraction augmented by isotopic substitution has been widely used to study the structure of hydrogen containing liquids as well as the structure of aqueous and other solvent systems. ${ }^{2,32-38}$ Given that different isotopes give different neutron scattering amplitudes, multiple measurements on the same system using different isotopomers produce different diffraction patterns, allowing for the interpretation of the structure and coordination around different sites within the liquid. Neutron diffraction on solutions yields the quantity, $F(Q)$, which is the sum of all Faber-Ziman partial structure factors, $S_{\alpha \beta}(Q)$, present in the sample each weighted by their composition $(c)$ and scattering intensity $(b), F(Q)$ $=\Sigma_{\alpha \beta} c_{\alpha} c_{\beta} b_{\alpha} b_{\beta}\left(S_{\alpha \beta}(Q)-1\right)$, where $Q$ is the magnitude of the change in momentum vector by the scattered neutrons $(Q=4 d \sin \theta / \lambda)$. Fourier transform of $S_{\alpha \beta}(Q)$ gives the respective atom-atom radial distribution functions (RDFs), $g_{\alpha \beta}(r)$, and integration of $g_{\alpha \beta}(r)$ gives the coordination number of atoms $\alpha$ around $\beta$ atoms between two distances, $r_{1}$ and $r_{2}{ }^{36,37}$

In order to extract site-specific information regarding acetone and DMSO as solutes in water, neutron scattering data were collected on samples of each of the binary systems at $298 \pm 2$ K. All measurements were obtained using the SANDALS diffractometer located at the ISIS Facility at the Rutherford Appleton Laboratory in the UK. For each sample measured the raw data for the samples as well as for the sample containers have been corrected for absorption, multiple scattering, and inelasticity effects, by using a program, GUDRUN, which is available at ISIS. ${ }^{39}$

Fully deuterated and protiated DMSO and acetone, as 
TABLE I. Acetone-water solutions measured by neutron diffraction.

\begin{tabular}{lc}
\hline \hline Sample & $1: 20$ acetone:water \\
\hline I & $\left(\mathrm{CD}_{3}\right)_{2} \mathrm{CO}: \mathrm{D}_{2} \mathrm{O}$ \\
II & $\left(\mathrm{CH}_{3}\right)_{2} \mathrm{CO}: \mathrm{HDO}$ \\
III & {$\left[\left(\mathrm{CH}_{3}\right)_{2} \mathrm{CO}\right]_{0.5}\left[\left(\mathrm{CD}_{3}\right)_{2} \mathrm{CO}\right]_{0.5}: \mathrm{HDO}$} \\
IV & $\left(\mathrm{CD}_{3}\right)_{2} \mathrm{CO}: \mathrm{H}_{2} \mathrm{O}$ \\
V & $\left(\mathrm{CH}_{3}\right)_{2} \mathrm{CO}: \mathrm{H}_{2} \mathrm{O}$ \\
& $1: 2$ acetone:water \\
I & $\left(\mathrm{CD}_{3}\right)_{2} \mathrm{CO}: \mathrm{D}_{2} \mathrm{O}$ \\
II & $\left(\mathrm{CH}_{3}\right)_{2} \mathrm{CO}: \mathrm{H}_{2} \mathrm{O}$ \\
III & $\left(\mathrm{CH}_{3}\right)_{2} \mathrm{CO}: \mathrm{D}_{2} \mathrm{O}$ \\
IV & {$\left[\left(\mathrm{CH}_{3}\right)_{2} \mathrm{CO}_{0.5}\left[\left(\mathrm{CD}_{3}\right)_{2} \mathrm{CO}\right]_{0.5}: \mathrm{HDO}_{0.5}: \mathrm{D}_{2} \mathrm{O}\right.$} \\
V & {$\left[\left(\mathrm{CH}_{3}\right)_{2} \mathrm{CO}_{0.5}\left[\left(\mathrm{CD}_{3}\right)_{2} \mathrm{CO}\right]_{0.5}: \mathrm{H}_{2} \mathrm{O}\right.$} \\
VI & {$\left[\left(\mathrm{CH}_{3}\right)_{2} \mathrm{CO}_{0.5}\left[\left(\mathrm{CD}_{3}\right)_{2} \mathrm{CO}\right]_{0.5}\right.$} \\
\hline \hline
\end{tabular}

well as ultrapure $\mathrm{D}_{2} \mathrm{O}$, were purchased from Sigma-Aldrich Chemical company and used without further purification and ultrapure $\mathrm{H}_{2} \mathrm{O}$ was obtained from a Millipore ${ }^{\circledR}$ purification apparatus. The samples were prepared by weight directly in the sample cans. Each of the samples measured were contained in flat plate cells with an internal thickness of $1 \mathrm{~mm}$ and a wall thickness of $1 \mathrm{~mm}$. The containers were constructed from a Ti/Zr null alloy metal, a special alloy, which results in negligible Bragg scattering from the container leading to a more tractable data analysis. All measured isotopomers of the acetone-water solutions and the DMSOwater solutions are listed in Tables I and II, respectively, and the densities for each concentration of DMSO and acetone in solution are listed in Table III. ${ }^{40,41}$

SANDALS is equipped with a transmission monitor which measures the total cross section of the sample being measured, $\sigma_{\text {trans }}$, as function of incident neutron energy. This total cross section was used to calculate the attenuation and multiple scattering corrections for the data, ensuring that the correct energy dependence of the corrections was included. The absolute level of each corrected $d \sigma / d \Omega$ was established by comparison with scattering from a standard vanadium sample where the cross section is known, and also in comparison with the corresponding theoretical values based the atomic composition. $^{42}$ In each case the measured level was within $15 \%$ of the expected value. Uncertainties of this magnitude can arise because of small uncertainties in the precise internal thickness of the sample container, and because the

TABLE II. DMSO-water solutions measured by neutron diffraction.

\begin{tabular}{lc}
\hline \hline Sample & $1: 20 \mathrm{DMSO}:$ water \\
\hline I & $\left(\mathrm{CD}_{3}\right)_{2} \mathrm{SO}: \mathrm{D}_{2} \mathrm{O}$ \\
II & $\left(\mathrm{CD}_{3}\right)_{2} \mathrm{SO}: \mathrm{HDO}$ \\
III & {$\left[\left(\mathrm{CH}_{3}\right)_{2} \mathrm{SO}\right]_{0.5}\left[\left(\mathrm{CD}_{3}\right)_{2} \mathrm{SO}\right]_{0.5}: \mathrm{HDO}$} \\
IV & $\left(\mathrm{CD}_{3}\right)_{2} \mathrm{SO}: \mathrm{H}_{2} \mathrm{O}$ \\
V & $\left(\mathrm{CH}_{3}\right)_{2} \mathrm{SO}: \mathrm{H}_{2} \mathrm{O}$ \\
& $1: 2 \mathrm{DMSO}_{\mathrm{D}_{2}}$ \\
I & $\left(\mathrm{CD}_{3}\right)_{2} \mathrm{SO}: \mathrm{D}_{2} \mathrm{O}$ \\
II & {$\left[\left(\mathrm{CH}_{3}\right)_{2} \mathrm{SO}_{0.5}\left[\left(\mathrm{CD}_{3}\right)_{2} \mathrm{SO}\right]_{0.5}: \mathrm{HDO}\right.$} \\
III & $\left(\mathrm{CH}_{3}\right)_{2} \mathrm{SO}: \mathrm{H}_{2} \mathrm{O}$ \\
\hline \hline
\end{tabular}

TABLE III. Densities for measured concentrations of DMSO and acetone in water.

\begin{tabular}{lc}
\hline \hline Concentration & Density $\left(\right.$ atoms $\left./ \AA^{3}\right)$ \\
\hline $1: 2$ acetone:water & 0.09112 \\
$1: 20$ acetone:water & 0.09845 \\
$1: 2$ DMSO:water & 0.08807 \\
$1: 20$ DMSO:water & 0.09645 \\
\hline \hline
\end{tabular}

theoretical level of the diffraction cross section at high $Q$, particularly for light hydrogen containing samples, is subject to a significant correction for inelasticity effects which can be estimated only approximately. Fortunately the contrast between hydrogen and deuterium in the diffraction cross sections is much larger than this uncertainty, as can be seen in the data shown below.

\section{EPSR ANALYSIS}

Empirical potential structure refinement (EPSR) was used to model the diffraction data collected on both the DMSO-water and acetone-water solutions. EPSR is a computational method for modeling disordered materials. ${ }^{43,44}$ It generates a three dimensional model consistent with a set of one-dimensional structure factor measurements. In EPSR a set of reference potentials are used to seed a Monte Carlo computer simulation of the system in question. These reference potentials, which include information on the expected molecular geometries as well as likely minimum approach distances between atoms plus effective charges on individual atoms where appropriate, are then perturbed interactively by the program until a structural model which is consistent with the diffraction data is obtained. ${ }^{31}$ It should be noted that a single EPSR model is constrained to fit all the isotopically distinct yet chemically identical diffraction patterns for each system at a particular concentration simultaneously.

For the EPSR simulations on both acetone and DMSO in water, a box of molecules was constructed at the appropriate concentration and density (Table III) of the measurements. The DMSO 1:20 solution the modeling box consisted of 24 DMSO molecules and 480 water molecules while in the acetone 1:20 solutions modeling box contained 500 water molecules and 25 acetone molecules. For the both the acetone and DMSO 1:2 concentrations the modeling box consisted of 167 solute molecules and 333 water molecules and the simulation box sizes were between 2.6 and $3.1 \mathrm{~nm}$. The reference potentials for this EPSR study are listed in Table IV. The initial potential for DMSO was modified from the P1 potential and the Rao-Singh potential for DMSO, ${ }^{38,45,46}$ which both use a unified atom potential for the methyl group. It should be noted that this is a different reference potential than used for the previous investigation of 1:2 DMSO water/solutions ${ }^{27}$ and is slightly modified from the case of pure DMSO. ${ }^{29}$ The initial potentials for acetone are FMHK $^{17,29}$ and SPC/E for water. ${ }^{47}$ It should be emphasized that the reference potentials are modified through the EPSR process to produce a structural model which is consistent with the measured diffraction data and as such slight differences will have a negligible impact on the resultant structure. 
TABLE IV. Reference potentials used for initial input into EPSR fits to diffraction data for acetone and DMSO (Refs. 17, 29, and 44-46). Note that in both this work and the previous pure acetone work (Ref. 29) the value of $\varepsilon$ for $\mathrm{C}_{m}$ used in the acetone reference potential is marginally different from its original value of $0.7065 \mathrm{~kJ} / \mathrm{mol}$ (Ref. 17). This error was not noticed until after both the previous simulations on pure acetone and the present simulations on acetone-water solutions were completed. Such a small change to the reference potential will not have any significant structural impact on the outcome of the EPSR analysis.

\begin{tabular}{lllc}
\hline \hline Acetone $^{\mathrm{a}}$ & $\varepsilon / \mathrm{kJ} \mathrm{mol}^{-1}$ & $\sigma / \AA$ & $q_{e}$ \\
\hline $\mathrm{H}$ & 0.0 & 0.0 & 0.0 \\
$\mathrm{C}_{m}$ & 0.7605 & 3.88 & -0.032 \\
$\mathrm{C}_{C}$ & 0.43932 & 3.75 & 0.566 \\
$\mathrm{O}$ & 0.878 & 2.96 & -0.502 \\
& & & \\
$\mathrm{DMSO}$ & $\mathrm{b}$ & & 0.0 \\
$\mathrm{H}$ & 0.0 & 0.0 & 0.0 \\
$\mathrm{C}$ & 0.7605 & 3.88 & 0.54 \\
$\mathrm{~S}$ & 0.84517 & 3.56 & -0.54 \\
$\mathrm{O}$ & 0.27614 & 2.94 & \\
Water $^{\mathrm{c}}$ & & & -0.8476 \\
$\mathrm{O}_{w}$ & 0.65000 & 3.166 & 0.4238 \\
$\mathrm{H}_{w}$ & 0.0 & 0.0 & \\
\hline \hline
\end{tabular}

${ }^{\mathrm{a}}$ Reference 17.

${ }^{\mathrm{b}}$ References 45 and 46.

${ }^{\mathrm{c}}$ Reference 47.

\section{RESULTS}

The measured diffraction data along with the EPSR fits to the data are shown in Figs. 2 and 3 for both concentrations of DMSO and acetone solutions, respectively, where the samples are labeled according to Tables I and II. The fits to the diffraction data are generally good, except at the low $Q$ values $\left(\sim 0.1-2 \AA^{-1}\right)$ in a few of the data sets. This is likely primarily due to an inadequate background correction for inelasticity effects, which are difficult to remove completely from samples containing hydrogen.

\section{A. Water-water RDFs}

Figure 4 shows the water-water RDFs from each of the solutions measured compared with those of pure water. The corresponding coordination numbers for each of these functions are shown in Table V. For the acetone-water 1:20 concentrations, the peaks in all of the RDFs in Fig. 4 are in approximately same positions as those for the pure water case, and the $g_{\mathrm{O}_{w} \mathrm{H}_{w}}(r)$ and $g_{\mathrm{H}_{w} \mathrm{H}_{w}}(r)$ functions are only slightly higher than the corresponding curves for pure water. Conversely, in the 1:20 DMSO-water solution the peak positions in each RDF in Fig. 4 are shifted to slightly higher $r$ compared to those of pure water. The coordination numbers at this concentration (Table V) are also similar to those for pure water.

In contrast to the 1:20 solutions, both 1:2 water solutions show quite different water-water RDFs when compared with pure water and with the more dilute solutions. Specifically, in each correlation the peaks are much higher in amplitude in the 1:2 solutions than for pure water. A large contribution to this increased amplitude can be attributed to "excluded volume effects" $" 48,49$ which arise because the local water density

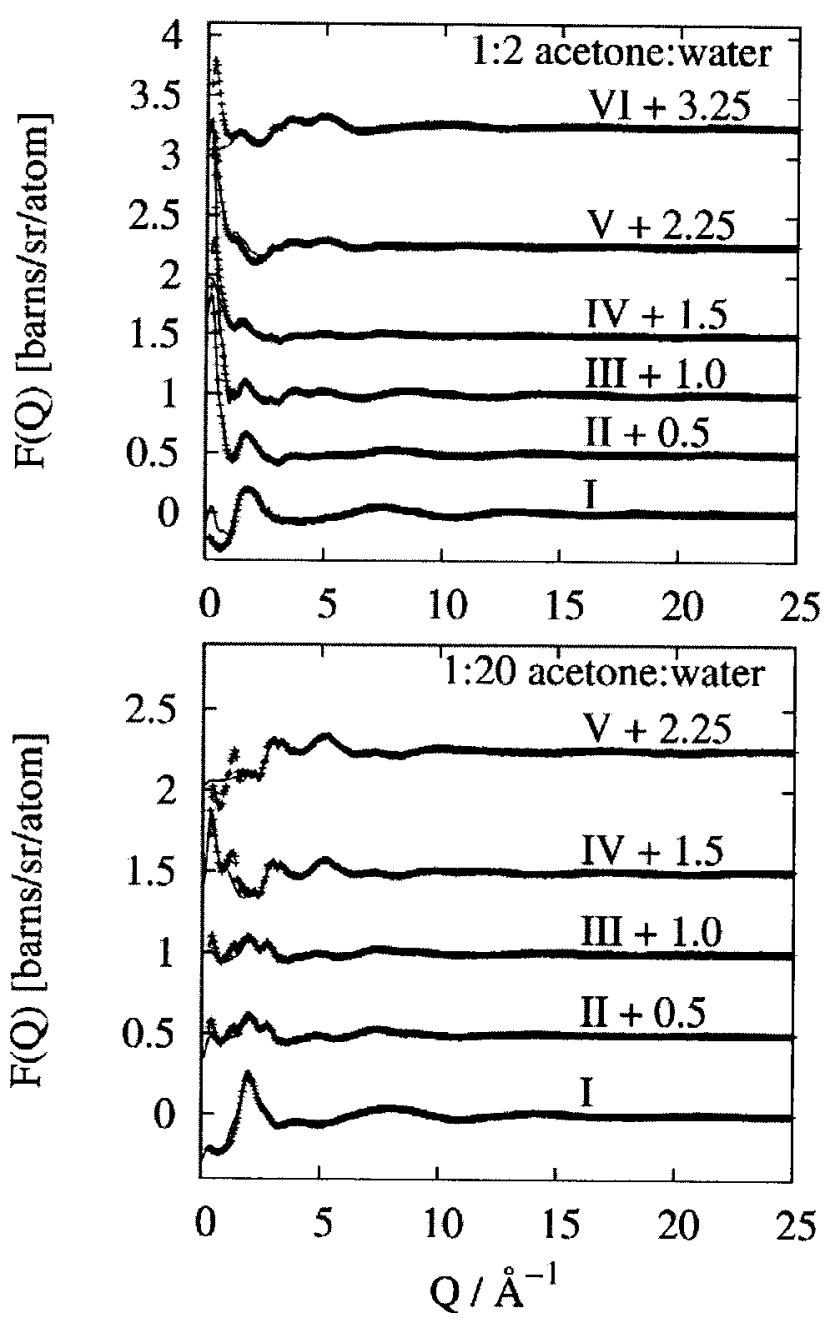

FIG. 2. Measured diffraction data (crosses) and subsequent EPSR fits to the diffraction data (solid lines) for 1:20 (bottom) and 1:2 (top) acetone-water solutions. The data are labeled according to Table I and in each case have been offset vertically for clarity.

in the immediate vicinity of any given water molecule is much higher than the average water density in the entire solution. ${ }^{50,51}$ This rise in the local density at short distances arises simply from the exclusion of water from the region occupied by the solute molecule and cannot be taken to indicate increased water structure. ${ }^{45}$ In the current simulations the excluded volume effects appear more pronounced than in the earlier work on DMSO:water. ${ }^{27}$ However, the waterwater coordination numbers shown here in Table $\mathrm{V}$ are in much closer agreement with previous molecular dynamics computer simulation $^{45}$ using different force fields for DMSO (P2) and water (SPC) than was found previously. ${ }^{27}$ It has been shown that the SPC/P2 model yields the most reasonable description of the mixture in terms of thermodynamic and transport properties. ${ }^{52}$ It is also of note that the excluded volume effect appears more marked for acetone-water (higher peaks at low r) compared to DMSO-water, suggesting that there is more segregation of water from solute in the acetone-water solutions compared to DMSO-water.

Notwithstanding this excluded volume effect on the peak heights, in both the 1:2 solutions the peak positions are at similar positions as those for pure water for the $g_{\mathrm{O}_{w} \mathrm{H}_{w}}(r)$ and 


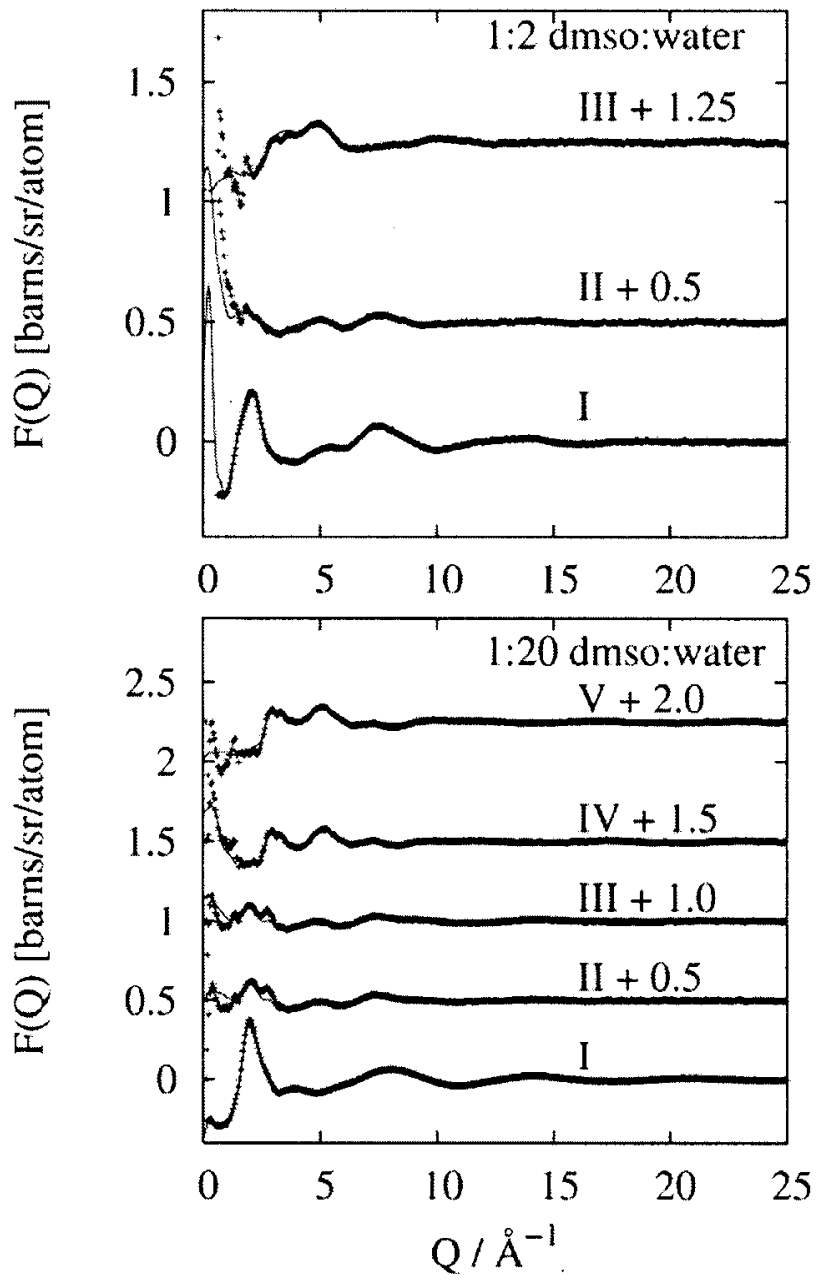

FIG. 3. Measured diffraction data (crosses) and subsequent EPSR fits to the diffraction data (solid lines) for 1:20 (bottom) and 1:2 (top) DMSO-water solutions. The data are labeled according to Table I and in each case have been offset vertically for clarity.

$g_{\mathrm{H}_{w} \mathrm{H}_{w}}(r) \mathrm{RDFs}$, while for the $g_{\mathrm{O}_{w} \mathrm{O}_{w}}(r) \mathrm{RDF}$ the first peak is at the same position as pure water but the second peak position is shifted to a higher $r$ value and is "flattened," compared with pure water, in both solutions. This "flattening" indicates that the second water-water coordination shell has been significantly perturbed by both solutes. This is not unexpected given the high concentration of solute in the 1:2 solutions but is not entirely consistent with the previous analysis of the experimental data for DMSO-water at this concentration. ${ }^{27}$ On the other hand, molecular dynamics results $^{27,45,50}$ do show a shift to larger $r$ albeit to a lesser extent than found here. This gives some indication of the limitations of diffraction data in being able by themselves to uniquely define the water structure in these solutions.

Although the water-water RDFs for the 1:2 solutions for both acetone and DMSO solutions look quite similar, the coordination numbers are different (Table V). The waterwater coordination numbers for the 1:2 DMSO solutions are roughly $20 \%-30 \%$ lower than the corresponding coordination numbers in the 1:2 acetone solutions. This variation is most pronounced in the $g_{\mathrm{O}_{w} \mathrm{H}_{w}}(r)$ coordination number which is 1.1 in the 1:2 DSMO water solution and 1.6 in the $1: 2$
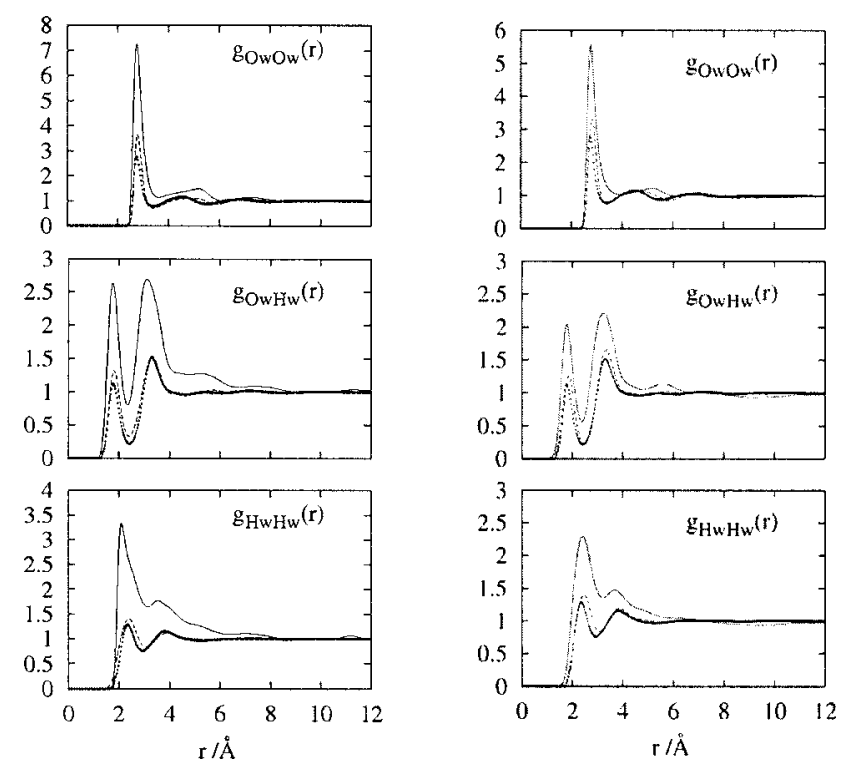

FIG. 4. Water-water RDFs for acetone-water solutions (left panel) and DMSO-water solutions (right panel) compared with pure water. For each of the depicted RDFs the solid line represents the 1:2 concentrations, the dashed line represents the 1:20 concentrations, and the circles represent pure water.

acetone-water solution, indicating that the water organization around the solutes in these solutions is different upon the addition of acetone or DMSO to pure water. For both solutes there is a reduction of all three water coordination numbers with increasing concentration, although DMSO/water solutions show the greatest decrease in water-water coordination. The greatly decreased water-water coordination in the 1:2 DMSO/water solutions indicates that the number of water molecules in the tetrahedral network is reduced as a result of water bonding more strongly to the DMSO than to itself in solution, ${ }^{25}$ compared to acetone at the same concentration. The same enhanced water bonding to DMSO compared to acetone would also explain the larger excluded volume effect in 1:2 acetone compared to 1:2 DMSO.

\section{B. Solute-water RDFs}

Figure 5 shows the acetone-water RDFs for both 1:2 and 1:20 acetone-water solutions. In all of the RDFs there are relatively small differences as a function of concentration and the only prominent hydrogen bonding between water

TABLE V. Coordination numbers for water-water correlations shown in Fig. 5 from DMSO-water and acetone-water solutions compared with pure water.

\begin{tabular}{|c|c|c|c|}
\hline & $\begin{array}{l}g_{\mathrm{O}_{w} \mathrm{O}_{w}}(r) \\
r=3.54 \AA\end{array}$ & $\begin{array}{l}g_{\mathrm{O}_{w} \mathrm{O}_{w}}(r) \\
r=2.40 \AA\end{array}$ & $\begin{array}{l}g_{\mathrm{H}_{w} \mathrm{H}_{w}}(r) \\
r=2.94 \AA\end{array}$ \\
\hline Pure water & $\sim 4.5-5$ & $\sim 1.8$ & $\sim 4.4$ \\
\hline $\begin{array}{l}1: 20 \\
\text { acetone:water }\end{array}$ & 5.2 & 1.9 & 5.2 \\
\hline $\begin{array}{l}\text { 1:20 } \\
\text { DMSO:water }\end{array}$ & 4.9 & 1.8 & 4.9 \\
\hline $\begin{array}{l}1: 20 \\
\text { acetone:water }\end{array}$ & 4.3 & 1.6 & 4.5 \\
\hline $\begin{array}{l}1: 2 \\
\text { DMSO:water }\end{array}$ & 3.4 & 1.2 & 3.3 \\
\hline
\end{tabular}



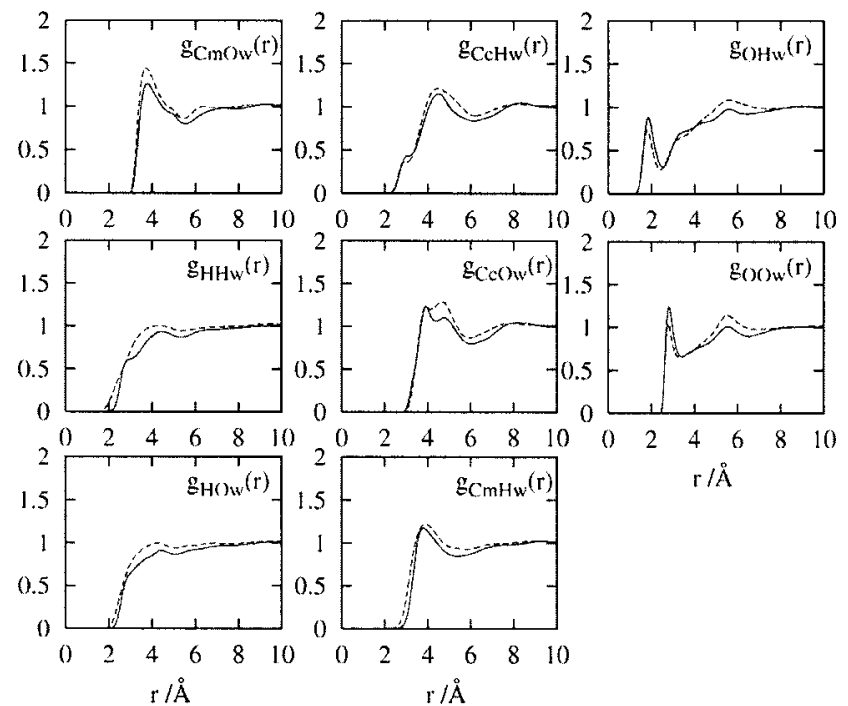

FIG. 5. Acetone-water RDFs from EPSR fits to the measured diffraction data for 1:20 (dashed lines) and 1:2 (solid lines) acetone-water solutions.

and acetone occurs as a hydrogen bond between water and the acetone oxygen atom. This hydrogen bond is evident from the $g_{\mathrm{OH}_{w}}(r)$ RDF which shows a peak at $\sim 1.9 \AA$ and the $g_{\mathrm{OO}_{w}}(r)$ RDF which has a peak at $\sim 2.8 \AA$. The coordination number for the $g_{\mathrm{OH}_{w}}(r)$ function is 1.2 at $2.40 \AA$ for the 1:20 acetone-water solution and 0.6 for the more concentrated acetone-water solution, showing a marked decrease in hydrogen bonding from water to acetone with increasing acetone content. It should be noted that the relative decrease in number of acetone-water hydrogen bonds $(\sim 50 \%)$ is larger than the corresponding decrease in water mole fraction, from 0.95 to 0.67 , between the two concentrations, suggesting that steric effects at high concentrations have a pronounced impact on the hydrogen bonding in these solutions.

Figure 6 shows the DMSO-water RDFs for both the DMSO-water solutions. In contrast to acetone-water solutions the RDFs for DMSO water are considerably different for the two different concentrations of DMSO. In most of the
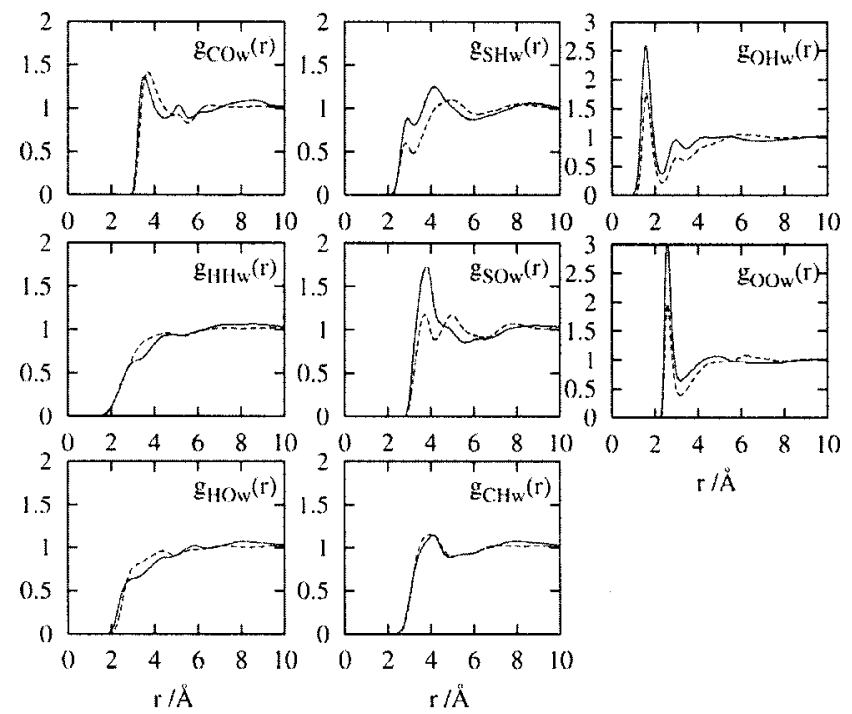

FIG. 6. DMSO-water RDFs from EPSR fits to the measured diffraction data for 1:20 (dashed lines) and 1:2 (solid lines) DMSO-water solutions.
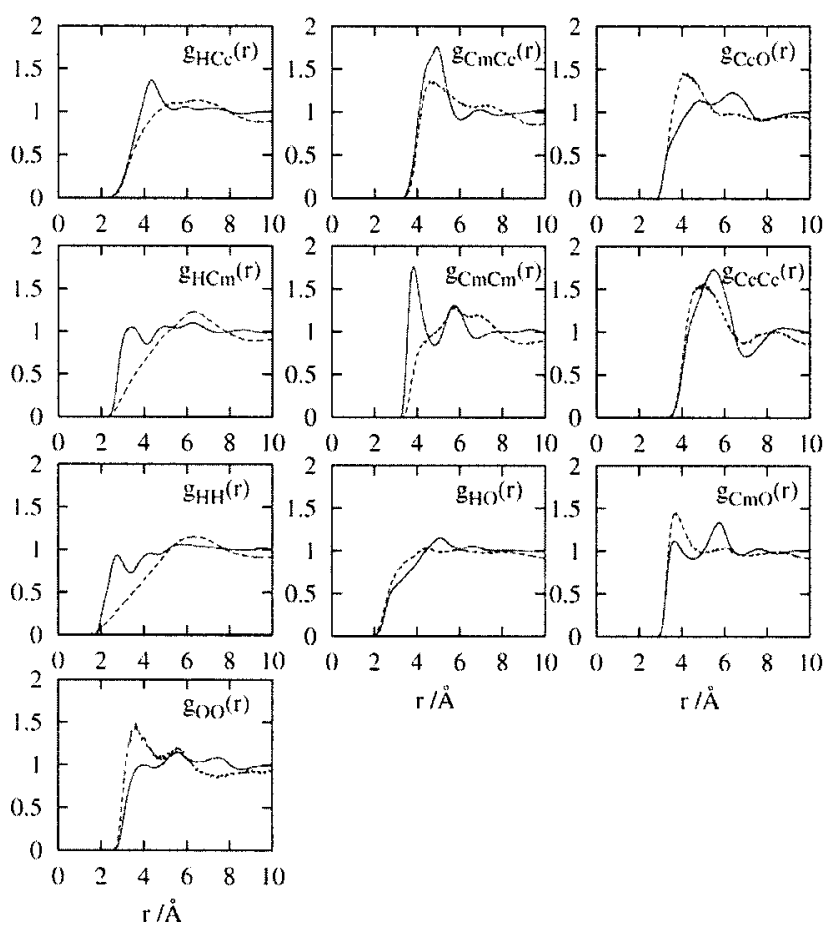

FIG. 7. Acetone-acetone RDFs from EPSR fits to the measured diffraction data for 1:20 (dashed lines) and 1:2 (solid lines) acetone-water solutions.

RDFs the interactions around the $\mathrm{S}=\mathrm{O}$ bond become considerably more pronounced with increasing concentrations of DMSO [the $g_{\mathrm{SO}_{w}}(r), g_{\mathrm{SH}_{w}}(r), g_{\mathrm{OO}_{w}}(r)$, and $g_{\mathrm{OH}_{w}}(r)$ all increase at short distances], while interactions with the methyl groups $\left[g_{\mathrm{CO}_{w}}(r), g_{\mathrm{CH}_{w}}(r), g_{\mathrm{HO}_{w}}(r)\right.$, and $\left.g_{\mathrm{HH}_{w}}(r)\right]$ all decrease slightly at the shorter distances. The most pronounced effects are in the $g_{\mathrm{SO}_{w}}(r)$ functions in Fig. 6. In the $g_{\mathrm{SO}_{w}}(r)$ RDF the two small relatively broad peaks at the lowest concentration become a single larger sharp peak at the shorter distance in the higher concentrations of DMSO.

Similar to the acetone-water RDFs (Fig. 5), the $g_{\mathrm{OO}_{w}}(r)$ and $g_{\mathrm{OH}_{w}}(r)$ in DMSO water both show sharp peaks at $\sim 3.5$ and $2 \AA$, respectively. Again, these distances are indicative of hydrogen bonding between the water molecules in the surrounding solvent to the DMSO solute molecules. The coordination numbers for the $g_{\mathrm{OH}_{w}}(r)$ function are 2.0 and 1.3 for the 1:20 and 1:2 solutions, respectively. As was the case with the acetone-water solution, the hydrogen bonding decreases with increasing concentration of solute, but the relative decrease is now closer to the change in water mole fraction. There are significantly more $\mathrm{O}-\mathrm{H}_{w}$ hydrogen bonds between DMSO and the surrounding water solvent in both DMSO-water solutions than seen for the corresponding concentrations of acetone in water.

\section{Solute-solute RDFs}

The solute-solute interactions for both acetone-water and DMSO-water solutions are reported in Figs. 7 and 8, respectively, for each concentration measured. Once again the trends with concentration are more marked in the DMSO solutions compared to acetone solutions. However, in both Figs. 7 and 8 there are increased methyl-methyl group associations at short distances in the more highly concentrated 

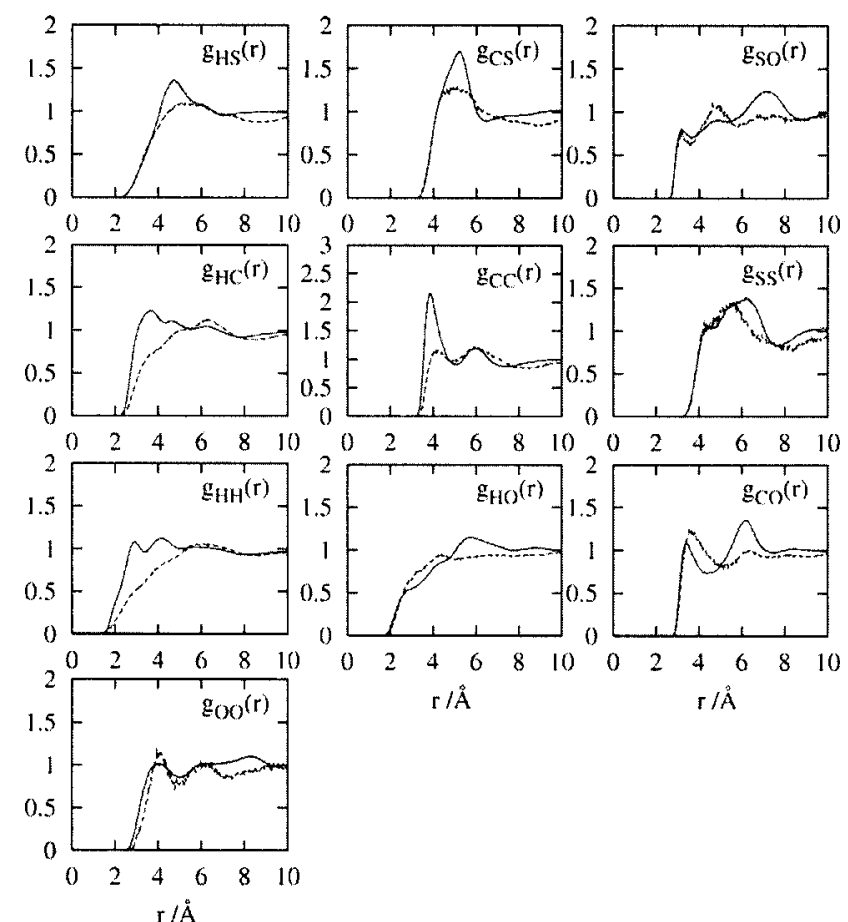

$r / \AA$

$\Gamma / \AA$ data for 1:20 (dashed lines) and 1:2 (solid line) DMSO-water solutions.

solutions $\left[g_{\mathrm{C}_{m} \mathrm{C}_{c}}(r), g_{\mathrm{C}_{m} \mathrm{C}_{m}}(r), g_{\mathrm{HC}_{m}}(r)\right.$, and $g_{\mathrm{HH}}(r)$ in acetone (Fig. 7); $g_{\mathrm{CS}}(r), g_{\mathrm{CC}}(r), g_{\mathrm{HC}}(r)$, and $g_{\mathrm{HH}}(r)$ in DMSO (Fig. 8)], while interactions between the $\mathrm{C}_{c}=\mathrm{O}$ bonds (acetone) and $\mathrm{S}=\mathrm{O}$ bonds (DMSO) and the methyl groups tend to decrease at these higher concentrations [viz., $g_{\mathrm{C}_{m} \mathrm{O}}(r)$ and $g_{\mathrm{HO}}(r)$ in acetone and $g_{\mathrm{CO}}(r)$ and $g_{\mathrm{HO}}(r)$ in DMSO]. Although the methyl-oxygen contacts decrease in the presence of a higher concentration of solute, the methyl-central atom interactions increase at these higher concentrations [viz., $g_{\mathrm{HC}_{c}}(r)$ and $g_{\mathrm{C}_{m} \mathrm{C}_{c}}(r)$ for acetone and $g_{\mathrm{HS}}(r)$ and $g_{\mathrm{CS}}(r)$ for DMSO]. This is mostly likely a consequence of the enhanced methyl-methyl interaction at this concentration as there is reduced interaction between the central atom and the $\mathrm{O}$ atom in both 1:2 solutions $\left[g_{\mathrm{C}_{c} \mathrm{O}}(r)\right.$ in acetone, $g_{\mathrm{SO}}(r)$ in DMSO]. The methyl-methyl interactions in DMSO solution are slightly more enhanced when compared to same interaction in acetone solution. This may be reflective of a stronger attraction of the $\mathrm{S}=\mathrm{O}$ bond for water, compared to the $\mathrm{C}=\mathrm{O}$ bond in acetone. Conversely, as described above, the excluded volume effects on the water-water correlations are stronger in acetone compared to water. In other words, because the DMSO attracts water more strongly than acetone, the net effect is a less segregated solution in DMSO-water compared to acetone-water. This effect is discussed in more detail below (Sec. IV E).

\section{Spatial density functions}

In addition to determining the RDFs from the EPSR model, some of the three dimensional spatial density functions (SDFs), which show the location of molecules or portions of molecules relative to one another were also determined. The SDFs are derived from a spherical harmonic expansion of the RDFs from the EPSR modeling box and

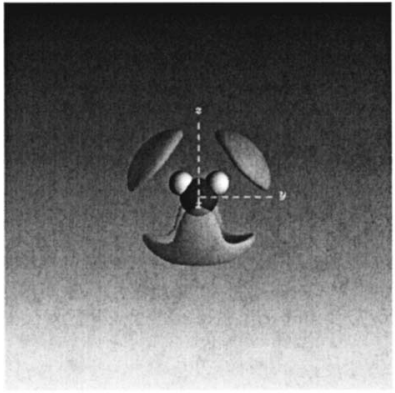

c) $1: 2$ acetone water 2 to $3.2 \AA$

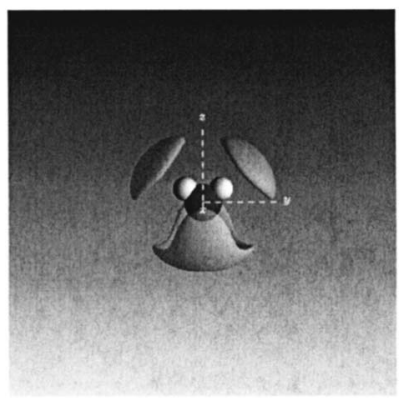

a) 1:20 acetone water 2 to $3.2 \AA$

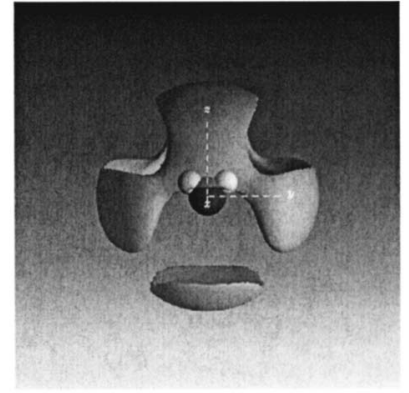

d) $1: 2$ acetone water 3.2 to $5 \AA$

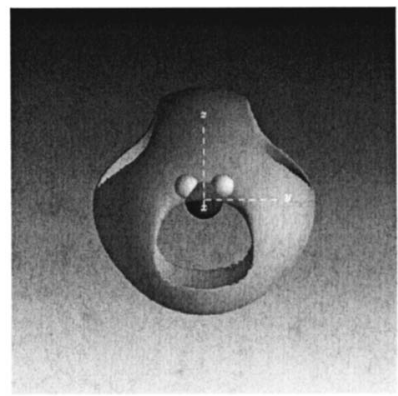

b) 1:20 acetone water 3.2 to $5 \AA$
FIG. 9. SDFs for water-water interactions. The bottom two panels (a) and (b) show the SDFs for the 1:20 solution and the top two panels (c) and (d) show the SDFs for the 1:2 solutions. The shells surrounding the central water molecule in panels (a) and (c), both from 2 to $3.2 \AA$, are shown at a contour level which encloses $60 \%$ of the water molecules in this distance range, while the shells in panels (b) and (d), from 3.2 to $5 \AA$, are shown at a contour level which encloses $50 \%$ of the water molecules in this distance range. In each panel the dimension of the plotting box is $16 \AA$.

provide a view of the liquid in three dimensions-details of the spherical harmonic expansion are given in detail elsewhere. ${ }^{29,37,54}$ Here, this expansion is shown for the waterwater interactions and for the solute water interactions.

\section{Water-water SDFs}

The water-water SDFs in all of the measured solutions were analyzed by placing the water oxygen atom at the center of the coordinate system with the dipole vector of the water molecule defining the $z$ axis and the hydrogen atoms lying in the $z y$ plane. The distribution of water molecules in the solution was probed in the space around this central water molecule, giving rise to the water-water SDFs. Figure 9 shows the water-water SDFs for both of the acetone-water solutions while Fig. 10 shows the same SDFs for both concentrations of DMSO in water. In all of the panels in both Figs. 9 and 10, the shell surrounding the water molecule on the central axis shows the distribution molecules in the range specified.

In both aqueous acetone solutions, the first coordination shell shows that the tetrahedral water coordination is not strongly disrupted in this distance range [Figs. 9(a)-9(c)] by the addition of acetone to water. This same first shell coordination is also observed at both concentrations of DMSOwater solutions [Figs. 10(a)-10(c)]. In fact, this water-water first coordination shell very similar to that is seen in pure water, with the exception that underneath the central water 

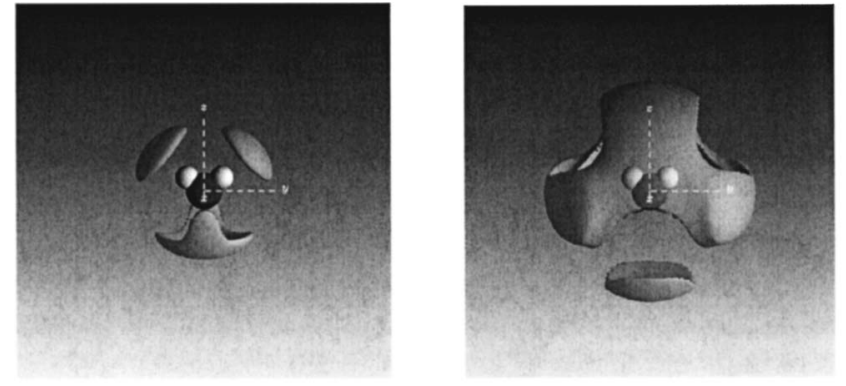

c) $1: 2 \mathrm{DMSO}$ water 2.0 to $3.2 \AA$

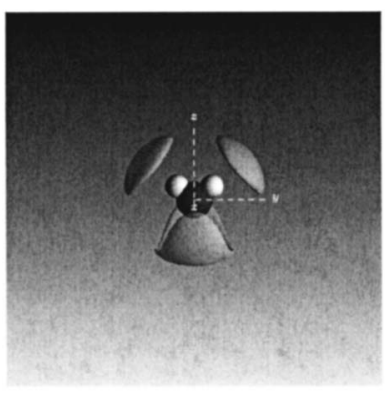

a) 1:20 DMSO water 2.0 to $3.2 \AA$ d) 1:2 DMSO water 3.2 to $5.0 \AA$

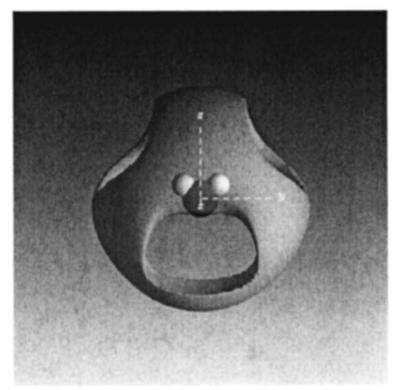

b) 1:20 DMSO water 3.2 to $5.0 \AA$

FIG. 10. SDFs for water-water interactions. The bottom two panels (a) and (b) show the SDFs for the 1:20 solution and the top two panels (c) and (d) show the SDFs for the 1:2 solutions. The shells surrounding the central water molecule in panels (a) and (c), both from 2 to $3.2 \AA$, are shown at a contour level which encloses $60 \%$ of the water molecules in this distance range, while the shells in panels (b) and (d), from 3.2 to $5 \AA$, are shown at a contour level which encloses $50 \%$ of the water molecules in this distance range. In each panel the dimension of the plotting box is $16 \AA$.

molecule, there is a growth of intensity in the $z y$ plane with increasing solute concentration compared to pure water. This indicates that there are probably increased trigonal water coordination as opposed to tetrahedral coordination at the higher solute concentrations. ${ }^{55}$ In the second coordination shell, the dilute concentration of both DMSO and acetone solutions show very similar SDFs where the most probable location of water molecules is similar to that of pure water, ${ }^{55}$ again with the exception of the band of density located underneath the oxygen atom below the $x y$ plane [Figs. 9(b) and $10(\mathrm{c})$ ]. In the higher 1:2 solutions the SDFs are slightly different in the second coordination shell for acetone-water compared with DMSO-water solutions [Figs. 9(d) and 10(d)] with the DMSO-water solution showing a slightly more diffuse distribution of water molecules in this second coordination shell.

\section{Acetone-water SDFs}

The SDFs for water around acetone were analyzed by placing the central carbon atom at the center of the coordinate system, with the $\mathrm{C}=\mathrm{O}$ bond defining the $z$ axis and with the methyl carbons lying in $z y$ plane below the $x y$ plane. Figure 11 shows the resultant SDFs from this operation for both 1:20 and 1:2 acetone-water solutions. Each panel shows the central planar acetone molecule while the surrounding shell shows the likely distribution of water molecules around this central acetone molecule over the specified distance ranges (2-4 and 4-6 $\AA$; the methyl hydrogen atoms have been omitted for clarity). These distance ranges were chosen
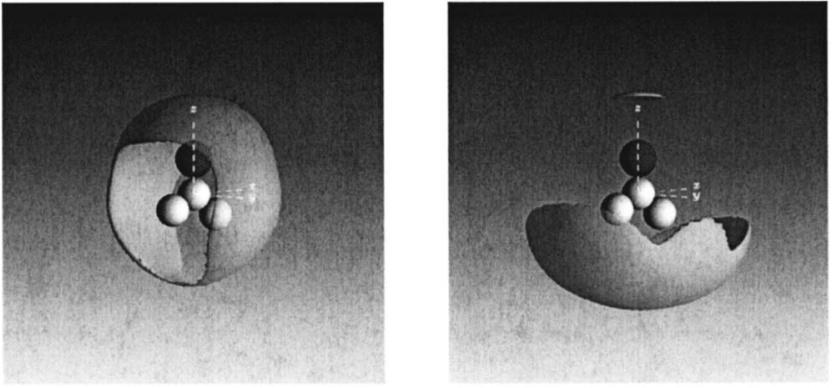

c) $1: 2$ acetone water 2 to $4 \AA$

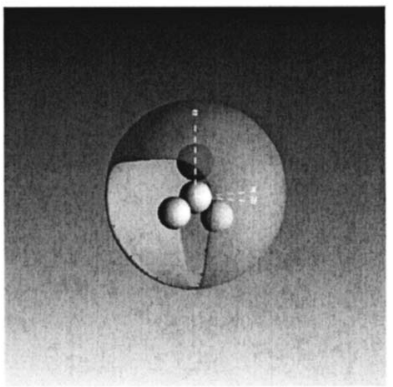

d) $1: 2$ acetone water 4 to $6 \AA$

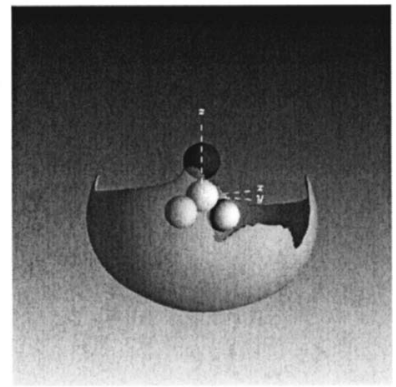

a) 1:20 acetone water 2 to $4 \AA$

b) 1:20 acetone water 4 to $6 \AA$

FIG. 11. SDFs for water around acetone. The bottom two panels (a) and (b) show the SDFs for the 1:20 solution and the top two panels (c) and (d) show the SDFs for the 1:2 solutions. The shells surrounding the central water molecule in panels (a) and (c), both from 2 to $4 \AA$, are shown at a contour level which encloses $80 \%$ of the water molecules in this distance range, while the shells in panels (b) and (d), from 4 to $6 \AA$, are shown at a contour level which encloses $25 \%$ of the water molecules in this distance range. In each panel the dimension of the plotting box is $16 \AA$.

as they can be roughly associated with the first and second hydration shell: $4 \AA$ is the approximate position of the maximum of the first peak in the $g_{\mathrm{C}_{c} \mathrm{O}_{w}}(r) \mathrm{RDF}$ at both concentrations (Fig. 6).

Inspection of this figure shows that at both concentrations the hydration shells are similar. In the closest hydration shell for concentrations [Figs. 11(a) and 11(c)] the SDFs are reminiscent of pure acetone SDFs: ${ }^{29}$ the band of water density in the surrounding hydration shell is either directly above the acetone molecule in the positive $z$ direction or located in bands in front and behind the plane of the acetone molecule along the central axis. In the second hydration shell [Figs. 11(b) and 11(d)], the SDFs for both solutions show the distribution of water molecules to be in a "cup" underneath the acetone molecule below the $x y$ plane, that is, around the methyl groups. This cup of density is somewhat larger in the 1:20 solution [Fig. 11(b)] than in the more concentrated case, indicating a more diffuse distribution of possible positions of water molecules at this concentration.

\section{DMSO-water SDFs}

In a similar manner to Fig. 11, Fig. 12 shows the SDFs for the DMSO-water solutions. In this case a DMSO molecule defines the central axes with the sulfur atom lying at the origin of the coordinate system and with the $\mathrm{S}=\mathrm{O}$ pointing along the positive $z$ axis. As opposed to acetone, the methyl carbons in DMSO are canted away from the $z y$ plane in the direction of the $x$ axis and lie slightly below the $x y$ plane reflecting the pyramidal symmetry of the molecule (the 

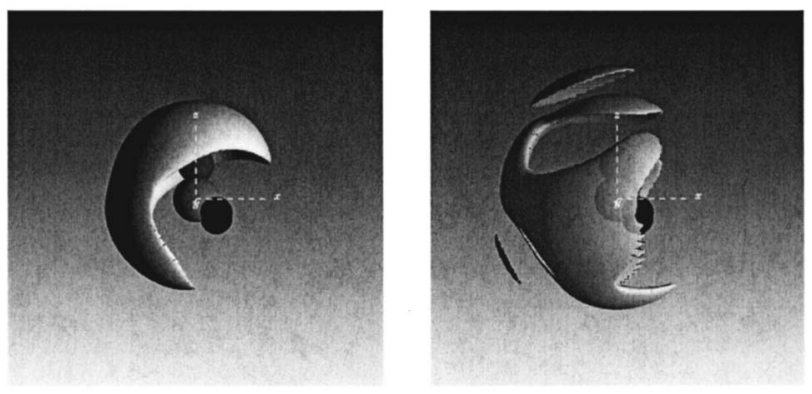

c) 1:2 DMSO water 2 to $4 \AA$

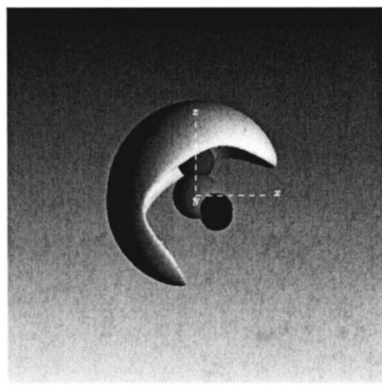

a) 1:20 DMSO water 2 to $4 \AA$

b) 1:20 DMSO water 4 to $6 \AA$

FIG. 12. SDFs for water around DMSO. The bottom two panels (a) and (b) show the SDFs for the 1:20 solution and the top two panels (c) and (d) show the SDFs for the 1:2 solutions. The shells surrounding the central DMSO molecule in panels (a) and (c), both from 2 to $4 \AA$, are shown at a contour level which encloses $80 \%$ of the water molecules in this distance range, while the shells in panels (b) and (d), from 4 to $6 \AA$, are shown at a contour level which encloses $25 \%$ of the water molecules in this distance range. In each panel the dimension of the plotting box is $16 \AA$.

methyl hydrogen atoms on the central molecule are omitted for clarity). Given that its molecular symmetry is different from that of acetone, the SDFs for water surrounding DMSO in solution are quite different from that of the acetone-water SDFs. Similar to the acetone-water solutions, the distance ranges for these SDFs were chosen to correspond with respect to the maxima of the $g_{\mathrm{SO}_{w}}(r)$ which occurs near $4 \AA$ (Fig. 6).

At both concentrations, in the closest hydration sphere surrounding DMSO [Figs. 12(a) and 12(c)], the highest probability of finding a water molecule is directly behind the DMSO molecule, in other words above the pyramid formed by the DMSO molecule itself. This density distribution of water is reflective of the SDF for pure DMSO ${ }^{29}$ suggesting that DMSO-DMSO interactions in pure DMSO liquid at this distance range are fully interchangeable with DMSO-water hydrogen bonds in solution. ${ }^{25}$ On the other hand, the second hydration shells about DMSO molecules are quite different from one another at differing concentrations of the solute in water. While the 1:20 concentration shows [Fig. 12(b)] a distribution, which is similar to the second sphere in pure DMSO, the 1:2 solution gives a drastically different SDF [Fig. 12(d)]. In the more concentrated case the distribution is again located above the DMSO pyramid and appears as another layer behind the first hydration shell shown in Fig. 12(c), indicating that the water layers between DMSO molecules are "stacked" along the negative $z y$ plane.
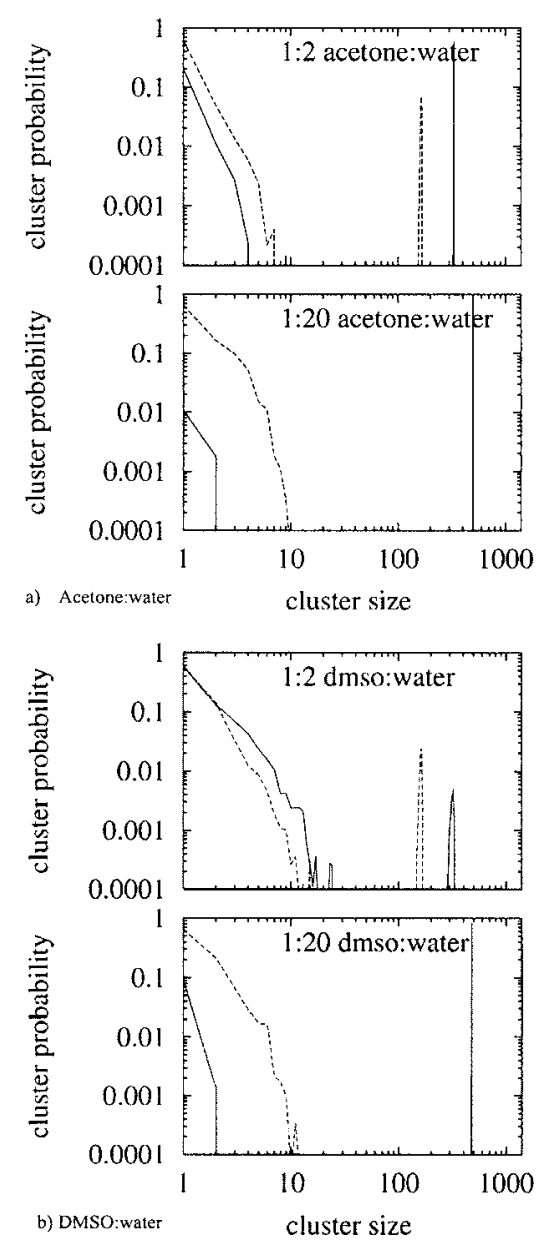

FIG. 13. Cluster size distribution for DMSO and acetone in aqueous solution. The dashed line shows the distribution of solute-solute clusters with cluster size, while the solid line shows the distribution of water-water clusters with cluster size. Panel (a) shows these cluster size distributions for the acetone-water solutions, while panel (b) shows the cluster size distributions for the DMSO-water solutions.

\section{E. Cluster analysis}

In order to determine the extent of clustering in acetonewater and DMSO-water solutions a cluster analysis was performed on the EPSR simulation boxes. Cluster analysis of aqueous solutions has been performed previously for several different solutes in water solutions. ${ }^{56-58}$ In the present analysis, water molecules were assigned to the same cluster if the $\mathrm{O}_{w}$ and $\mathrm{H}_{w}$ atoms on different water molecules were in the distance range from $r_{\min }=1.4 \AA$ to $r_{\max }=2.5 \AA$. DMSO and acetone molecules were considered to be in the same cluster if the central atoms on different molecules $\left(\mathrm{C}_{c}\right.$ in acetone and $S$ in DMSO) were in the distance range of 2.0-5.5 $\AA$. The results of the cluster size distribution are shown in Fig. 13(a) for acetone at both concentrations and Fig. 13(b) for DMSO at both concentrations.

For both 1:20 solutions, it is seen that the solute clusters (dashed lines) reach up to sizes of approximately nine molecules per cluster (acetone) and seven molecules per cluster (DMSO). Given that the cluster distribution decreases rapidly with increasing cluster size, the difference between these numbers is probably significant; specifically acetone has a greater tendency to cluster than DMSO. A similar trend is 
observed in the more concentrated solutions where a spike in the cluster distribution of the solutes appears near 160 molecules, which is close to the number of solute molecules in each box (167). This spike in the solute-solute cluster distributions indicates that the largest solute cluster in the box is fully percolating. It is notable that the height of this peak is lower for DMSO compared to acetone $(\sim 0.5$ for DMSO compared to $\sim 0.7$ for acetone).

These trends in the solute clusters are mirrored by equivalent trends in the water clusters (solid lines in Fig. 13). At all concentrations in both solutions the water clusters are percolating, as witnessed by the large spikes at cluster sizes corresponding closely to the number of water molecules in the simulation box (333 water molecules for 1:2 and 500 for 1:20 for both acetone and DMSO solutions). The effect is most pronounced for the 1:2 concentrations, where for DMSO small clusters extending up to sizes of around 22 water molecules per cluster are observed, while for acetone in aqueous solution, the small water clusters contain only up to approximately four water molecules per cluster. On the other hand, the height of the sharp spike near 333 water molecules per cluster is $\sim 0.6$ for acetone $1: 2$ solutions, but only $\sim 0.01$ for DMSO 1:2 solutions, and markedly broader in DMSO than for acetone. These trends fit in well with the differences in the extent of excluded volume effects between the two solutes as seen in Sec. IV A.

\section{DISCUSSION}

The local water structure found in all of the solutions measured indicates that the bulk water tetrahedral local coordination is preserved in these solutions at all concentrations. Although the water-water coordination numbers are reduced with respect to pure water in each of the present solutions, inspection of the water-water RDFs (Fig. 4) shows the first peaks in each case in the same location as those of pure water or shifted only slightly. The solution RDFs generally show greater intensity than the pure water peaks most likely due to excluded volume effects, ${ }^{48-51}$ but there is little movement in the peak positions, except for Ow-Ow second peak as discussed below. Moreover the water-water SDFs for all of the solutions measured show the tetrahedral coordination in the nearest-neighbor shell of the bulk water network to be virtually identical to the nearest-neighbor shell in pure water. $^{44}$

The decrease in water-water coordination with increasing amounts of DMSO has already been noted in previous neutron diffraction studies where in these investigations it was observed that although the number of water-water hydrogen bonds has decreased the local tetrahedral coordination is preserved. ${ }^{21,27}$ The results presented here for DMSO and for acetone are consistent with that view. That these water-water RDFs are reflective of a largely preserved local bulk waterlike coordination can be compared with studies on solutes in water which greatly perturb this structure as is the case with concentrated salts in solution. ${ }^{59,60}$

Although the bulk water local coordination appears to be preserved in all of the measured solutions, the water-water coordination numbers for acetone-water solutions compared with DMSO-water solutions are quite different (Table V). In comparing acetone with DMSO both concentrations of acetone solutions show higher water-water coordination than for DMSO solutions. This indicates that in acetone-water solutions the water is more "segregated" than is the case for DMSO in water. Water segregation has also been indicated in several computer simulation and spectroscopy studies in acetone-water solutions. ${ }^{18,61,62}$ Similar studies on DMSOwater solutions do not show any obvious water clustering, but rather indicate that at higher concentrations of DMSO in aqueous solutions there is a large number of DMSO-water complexes formed, ${ }^{63-65}$ and it has been postulated that these DMSO-water clusters are responsible for the nonideal behavior observed for 1:2 DMSO-water solutions. ${ }^{45,53,64,65}$

With respect to the water network itself, in both 1:20 solutions, the second peak in the $g_{\mathrm{O}_{w} \mathrm{O}_{w}}(r) \mathrm{RDF}$ is at about the same position, $\sim 4.5 \AA$, as in the case of bulk water, Fig. 4 (top panels), while at the 1:2 concentration, this peak has clearly moved outward toward $\sim 5.2 \AA$, indicating a possible break up of the usual water network. The water-water SDFs (Figs. 9 and 10), however, indicate that this is not the case given that the first water-water coordination shell is similar in all measured solutions and is indeed almost identical to that of pure water. ${ }^{55}$ This result is consistent with a recent commentary, ${ }^{66}$ namely, that the shape and position of the second peak in $g_{\mathrm{O}_{w} \mathrm{O}_{w}}(r)$ tends to exaggerate rather minor changes in the three-dimensional arrangement of water molecules.

The assertion that water-water correlations are more prominent in acetone-water solutions compared to DMSO solutions especially at the higher concentrations is additionally supported by the observation that there are fewer acetone-water hydrogen bonds in either solution when compared with the corresponding DMSO-water solutions. In the DMSO-water system the coordination number of $\mathrm{H}_{w}$ about $\mathrm{O}$ (the DMSO oxygen atom) at the 1:20 concentration is 2.2 while the same coordination number in acetone is only 1.2 at the same concentration. The $\mathrm{O}-\mathrm{H}_{w}$ coordination number decreases in both cases with increasing concentration of solute, namely, for 1:2 acetone-water solutions the $\mathrm{O}-\mathrm{H}_{w}$ coordination number is 0.6 while in 1:2 DMSO-water solutions this number is still quite high at 1.3.

The trends associated with the differences in hydration between acetone and DMSO at different concentrations are mirrored in the cluster distributions (Fig. 13). For acetone, a greater tendency to form distinct water-water and acetoneacetone clusters is observed at both concentrations, whereas in DMSO the degree of both water-water and DMSO-DMSO cluster formations is reduced in comparison. This reduced segregation in DMSO appears to be associated with the significantly enhanced hydration of the oxygen atom on DMSO compared to the same bond in acetone (compare Fig. 6 with Fig. 5) indicating the possible formation of DMSO-water complexes. ${ }^{45,65}$ This is similar to what was determined both in previous neutron diffraction simulation studies and independent MD simulation studies of 1:2 DMSO-water solutions, where in both cases it was concluded that structure of this solution is dominated by DMSO-water interactions. ${ }^{27,45}$ At the same time the water-water hydrogen bonds in DMSO 
are markedly reduced compared to acetone at the same concentration (Table V), which again indicates that DMSO bonds to water much more strongly than acetone, causing the DMSO-water solution to appear less segregated than acetone in solution.

These observations provide a possible structural explanation for the extremes of excess mixing enthalpy observed for DMSO in solution at the $1: 2$ concentration, ${ }^{25,28}$ namely, because water hydrates DMSO more strongly than it hydrates acetone, there is a corresponding much larger release of enthalpy when the DMSO solution is made compared to acetone. The trends in the solute-water SDFs seen in DMSO (Fig. 12) compared to acetone (Fig. 11) further support this interpretation. Additionally this conclusion is sustained by a recent study of methanol in solution as a function of lowered temperature and increased pressure where it was seen that increased segregation of the solute and solvent went hand in hand with overall better mixing of the solvent and solute, as determined from the estimated excess entropies of mixing. ${ }^{67}$ This can only occur if the clusters are sheet- or chainlike, so that there is a large surface area available where the solute and solvent in the different clusters can mix. Specifically there is no marked decrease in the water-methyl group interactions in the higher concentrations of acetone, as occurs in the case of DMSO [See Figs. 11(d) and 12(d)]. At the same time enhanced attraction of DMSO for water compared to acetone suppresses the buildup of segregated DMSO or water clusters in solution.

One interesting observation is based on the experimentally determined enthalpy and Gibbs free energy of mixing for DMSO and acetone. ${ }^{24,28,67}$ The excess entropy of mixing is $-1.7 \mathrm{~kJ} / \mathrm{mol}$ for DMSO:water at a $1: 2$ mole ratio, while for acetone it is higher at $-0.425 \mathrm{~kJ} / \mathrm{mol}$. At first sight it may seem that these numbers are contrary to our observation here that acetone is more segregated than DMSO in solution. However, the entropy calculation requires more knowledge of the topology of the clusters than just their size. If the clusters are more sheet- or chainlike the entropy will be higher due to mixing at the large surface of the cluster. We suggest that this is what may be occurring in the case of acetone in solution. On the other hand DMSO, which apparently has a stronger $\mathrm{O} \cdots \mathrm{H}_{w}$ bond, pulls water away from the methyl groups. This tendency in the DMSO solutions, together with the pyramidal molecular geometry, would encourage more compact globularlike clusters, containing possibly both DMSO and water, ${ }^{25,45,65}$ with correspondingly reduced interfacial regions, thus lowering the overall translational entropy. Unfortunately the present neutron diffraction data do not extend to low enough $Q$ values to be able to test these hypotheses conclusively.

It is clear in this reanalysis of the earlier 1:2 DMSOwater diffraction data that the EPSR analysis of the total diffraction scattering cross sections shown here, although having many qualitative characteristics in common, are not identical to what was extracted previously based on the composite radial distribution functions. Part of this difference will arise from fitting in the measured reciprocal space, $Q$, as opposed to the previous real space analysis, which can only be reached from the experiment by direct Fourier transform.
Hence, the present analysis is a more direct interpretation of the measured data. Moreover, the previous analysis removed the intramolecular scattering functions prior to analysis whereas the present analysis includes the molecular structure as part of the structure refinement, with the molecular quantum zero point disorder modeled in a realistic manner. ${ }^{68}$ This can make a difference because of the overlap between intraand intermolecular distances. Nonetheless, there is no quantitative way of distinguishing between the two interpretations, and differences between the two results are indicative of uncertainties inherent in trying to extract structural information from what are relatively complex solution scattering data. The main achievement here is the extraction of qualitative trends for different solute geometries and solute concentrations under otherwise identical experimental and simulation conditions. These trends are unlikely to be affected by quantitative differences between different models.

\section{CONCLUSIONS}

The terms "structure breaker" and "structure maker" with regard to the effect of solute molecules on water have often been used to characterize the effects of different solutes when added to bulk water. This study supports previous work on DMSO/water solutions, ${ }^{21}$ namely, it appears that these terms are likely too general to be useful in a quantitative analysis of structure in water solutions. Specifically, the water tetrahedral coordination appears to be intact in both DMSO- and acetone-water solutions at both concentrations. At the 1:20 concentrations for both solutes, the water network remains largely intact, but at 1:2 it appears to be slightly disrupted in the second coordination shell, though this is most likely an effect of decreased water concentration rather than a "structure making" or "breaking" effect. Although the total number of water-water hydrogen bonds are decreased in the present solutions compared to pure water, this decrease is largely an effect of steric restrictions on the number of water molecules that can hydrate the solute rather than due to any fundamental change in the local order of the bulk water network itself, in agreement with previous findings. ${ }^{18,21,27,45}$

In addition, DMSO forms significantly more hydrogen bonds via the DMSO oxygen atom with the surrounding water solvent than the equivalent site on acetone does at the same concentrations, which may provide structural evidence for the nonideal behavior of DMSO-water solutions at this concentration. In particular, in the highest concentration of DMSO, ${ }^{27}$ the water-water coordination appears to be stronger in that it effectively draws water away from the methyl groups, giving DMSO the possibility of forming relatively tightly bound globular clusters with water. In both acetonewater concentrations, water and acetone have a greater tendency to segregate into distinct water-water and acetoneacetone clusters compared with DMSO, but this greater segregation in acetone:water compared to DMSO:water could at the same time be reconciled with better overall solute-solvent mixing if the clusters are primarily sheet- or chainlike rather than globular in nature. 


\section{ACKNOWLEDGMENTS}

The authors thank the US-National Science Foundation for support to one of the authors (S.E.M.) under Award No. OISE-0404938 and for support to another author (A.L.) through Award Nos. CHE-0211626 and CHE-0512131.

${ }^{1}$ A. K. Soper, Science 297, 1288 (2002).

${ }^{2}$ J. L. Finney, D. T. Bowron, and A. K. Soper, J. Phys.: Condens. Matter 12, A123 (2000).

${ }^{3}$ D. T. Bowron, J. L. Finney, and A. K. Soper, J. Phys. Chem. B 102, 3551 (1998).

${ }^{4}$ J. Turner, J. L. Finney, J. P. Bouquiere, G. W. Neilson, S. Cummings, and J. Bouillot, Physica B \& C 136, 260 (1986).

${ }^{5}$ J. L. Finney, A. K. Soper, and J. Z. Tuner, Pure Appl. Chem. 65, 2521 (1993).

${ }^{6}$ J. Turner, A. K. Soper, and J. L. Finney, Mol. Phys. 77, 411 (1992).

${ }^{7}$ J. Turner, J. L. Finney, and A. K. Soper, Z. Naturforsch., A: Phys. Sci. 46, 73 (1991).

${ }^{8}$ J. Turner, A. K. Soper, and J. L. Finney, Mol. Phys. 70, 679 (1990).

${ }^{9}$ J. L. Finney, A. K. Soper, and J. Turner, Proceedings of the 12th International Conference Prop. Water Steam, 1995, p. 468.

${ }^{10}$ L. C. G. Freitas, J. M. M. Cordeiro, and F. L. L. Garbajo, J. Mol. Liq. 79, 1 (1999)

${ }^{11}$ A. Idrissi, F. Sokolic, and A. Perera, J. Chem. Phys. 112, 9479 (2000).

${ }^{12}$ A. Idrissi, S. Longelin, and F. Sokolic, J. Phys. Chem. B 105, 6004 (2001).

${ }^{13}$ J.-J. Max and C. Chapados, J. Chem. Phys. 119, 5632 (2003).

${ }^{14}$ J.-J. Max and C. Chapados, J. Chem. Phys. 120, 6625 (2003).

${ }^{15}$ J. Richardi, P. H. Fries, R. Fischer, S. Rast, and H. Krienke, Mol. Phys. 93, 925 (1998).

${ }^{16}$ Y. Takebayashi, S. Yoda, T. Sugeta, K. Otake, T. Sako, and M. Nakahara, J. Chem. Phys. 120, 6100 (2004)

${ }^{17}$ M. Ferrario, M. Haughney, I. R. McDonald, and M. L. Klein, J. Chem. Phys. 93, 5156 (1990).

${ }^{18}$ D. S. Venables and C. A. Schmuttenmaer, J. Chem. Phys. 113, 11222 (2000).

${ }^{19}$ A. Brandt, S. Magazu, A. Mangione, F. Migliardo, and B. G. Vertessy, Appl. Phys. A: Mater. Sci. Process. 74, S457 (2002).

${ }^{20}$ P. E. Mason, G. W. Neilson, J. E. Enderby, M. L. Saboungi, C. E. Dempsey, A. D. MacKerell, and J. W. Brady, J. Am. Chem. Soc. 126, 11462 (2004)

${ }^{21}$ A. K. Soper and A. Luzar, J. Chem. Phys. 97, 1320 (1992).

${ }^{22}$ S. W. Jacob, Pharmacology of DMSO in Dimethyl Sulfoxide (Dekker, New York, 1971).

${ }^{23}$ J. E. Lovelock and M. W. H. Bishop, Nature (London) 183, 1394 (1959).

${ }^{24}$ J. M. Cowie and P. M. Toporowski, Can. J. Chem. 39, 2240 (1961).

${ }^{25}$ A. Luzar, J. Chem. Phys. 91, 3603 (1989).

${ }^{26}$ A. Vishnyakov, A. P. Lyubartsev, and A. Laaksonen, J. Phys. Chem. A 105, 1702 (2001).

${ }^{27}$ A. K. Soper and A. Luzar, J. Phys. Chem. 100, 1357 (1996).

${ }^{28}$ E. Tommila and A. Pajunen, Suom. Kemistil. B41, 172 (1969); M. F. Fox and K. P. Whittingham, J. Chem. Soc., Faraday Trans. 75, 1407 (1974); J. Kenttammaa and J. J. Lindberg, Suom. Kemistil. B33, 32 (1960)
${ }^{29}$ S. E. McLain, A. K. Soper, and A. Luzar, J. Chem. Phys. 124, 074502 (2006).

${ }^{30}$ A. K. Soper, Chem. Phys. 202, 295 (1996).

${ }^{31}$ A. K. Soper, Phys. Rev. B 72, 104204 (2005).

${ }^{32}$ A. K. Soper, Z. Phys. B: Condens. Matter 276-278, 12 (2000).

${ }^{33}$ H. E. Fischer, A. C. Barnes, and P. S. Salmon, Rep. Prog. Phys. 69, 223 (2006).

${ }^{34}$ J. F. C. Turner and A. K. Soper, Polyhedron 23, 2975 (2004)

${ }^{35}$ E. C. Hulme, A. K. Soper, S. E. McLain, and J. L. Finney, Biophys. J. 91, 2371 (2006).

${ }^{36}$ H. Thompson, J. C. Wasse, N. T. Skipper, S. Hayama, D. T. Bowron, and A. K. Soper, J. Am. Chem. Soc. 125, 2572 (2003).

${ }^{37}$ S. E. McLain, A. K. Soper, and A. Watts, J. Phys. Chem. B 110, 21251 (2006).

${ }^{38}$ A. Luzar, A. K. Soper, and D. Chandler, J. Chem. Phys. 99, 6836 (1993).

${ }^{39}$ A. K. Soper, W. S. Howells, and A. C. Hannon, Report No. RAL-89-046, 1989.

${ }^{40}$ M. Dizechi and E. Marscall, J. Chem. Eng. Data 27, 358 (1982).

${ }^{41}$ D. J. Pruett and L. K. Felker, J. Chem. Eng. Data 30, 452 (1985).

${ }^{42}$ V. F. Sears, Neutron News 3, 29 (1992).

${ }^{43}$ A. K. Soper, Mol. Phys. 99, 1503 (2001).

${ }^{44}$ A. K. Soper, Chem. Phys. 258, 121 (2000).

${ }^{45}$ A. Luzar and D. Chandler, J. Chem. Phys. 98, 8160 (1993)

${ }^{46}$ B. G. Rao, and U. C. Singh, J. Am. Chem. Soc. 112, 3808 (1990).

${ }^{47}$ H. J. C. Berendsen, J. R. Grigera, and T. P. Straatsma, J. Phys. Chem. 91, 6269 (1987)

${ }^{48}$ A. K. Soper, J. Phys.: Condens. Matter 9, 2399 (1997).

${ }^{49}$ A. K. Soper, F. Bruni, and M. A. Ricci, J. Chem. Phys. 109, 1486 (1998).

${ }^{50}$ I. I. Vaisman and M. L. Berkowitz, J. Am. Chem. Soc. 114, 7889 (1992).

${ }^{51}$ B. M. Ladanyi and M. S. Skaf, Annu. Rev. Phys. Chem. 44, 335 (1993).

${ }^{52}$ M. Charalis and J. Samios, J. Mol. Liq. 98, 399 (2002).

${ }^{53}$ J. T. Cabral, A. Luzar, J. Teixeira, and M. C. Bellissent-Funel, J. Chem. Phys. 113, 8736 (2000).

${ }^{54}$ C. G. Gray and K. E. Gubbins, Theory of Molecular Liquids Vol I: Fundamentals (Oxford University Press, New York, 1984).

${ }^{55}$ A. K. Soper and M. A. Ricci, Phys. Rev. Lett. 84, 2881 (2000).

${ }^{56}$ L. Dougan, S. P. Bates, R. Hargreaves, J. P. Fox, J. Crain, J. L. Finney, V. Reat, and A. K. Soper, J. Chem. Phys. 121, 6456 (2004).

${ }^{57}$ S. E. McLain, A. K. Soper, A. E. Terry, and A. Watts, J. Phys. Chem. B 111, 4568 (2007)

${ }^{58}$ A. Oleinikova, I. Brovchenko, A. Geiger, and B. Guillot, J. Chem. Phys. 117, 3296 (2002)

${ }^{59}$ A. Botti, F. Bruni, S. Imberti, M. A. Ricci, and A. K. Soper, J. Chem. Phys. 119, 5001 (2003)

${ }^{60}$ R. Leberman and A. K. Soper, Nature (London) 378, 364 (1995).

${ }^{61}$ S. Weerasinghe and P. A. Smith, J. Chem. Phys. 118, 10663 (2003).

${ }^{62}$ A. Perera and F. Sokolic, J. Chem. Phys. 121, 11272 (2004).

${ }^{63}$ C. Nieto-Draghi, J. B. Avalos, and B. Rousseau, J. Chem. Phys. 119, $4782(2003)$

${ }^{64}$ B. Kirchner and M. Reiher, J. Am. Chem. Soc. 124, 6206 (2002).

${ }^{65}$ I. A. Borin and M. S. Skaf, J. Chem. Phys. 110, 6412 (1999).

${ }^{66}$ P. E. Mason and J. W. Brady, J. Phys. Chem. B 111, 5669 (2007).

${ }^{67}$ B. A. Coomber and C. J. Wormald, J. Chem. Thermodyn. 8, 793 (1976); M. A. Villamanan and H. C. Van Ness, J. Chem. Eng. Data 29, 429 (1984).

${ }^{68}$ L. Dougan, A. K. Soper, J. L. Finney, and J. Crain (unpublished) 